\title{
IFNG, FCER1A, PCDHB10 expression as a new potential marker of efficacy in grass pollen allergen-specific immunotherapy
}

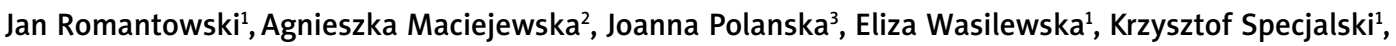 \\ Marta Chełminska ${ }^{1}$, Ewa Jassem ${ }^{1}$, Marek Niedoszytko ${ }^{1}$
}

${ }^{1}$ Department of Allergology, Medical University of Gdansk, Gdansk, Poland

${ }^{2}$ Department of Forensic Medicine, Medical University of Gdansk, Gdansk, Poland

${ }^{3}$ Faculty of Automatic Control, Electronics, and Computer Science, Silesian University of Technology, Katowice, Poland

Adv Dermatol Allergol 2021; XXXVIII (4): 665-672

DOI: https://doi.org/10.5114/ada.2021.108925

\begin{abstract}
Introduction: Allergen-specific immunotherapy (AIT) is the core treatment in allergic rhinitis and asthma. Although widely used, some patients do not benefit from treatment and there is no efficacy objective marker.

Aim: To define the profile of gene transcripts during the build-up phase of AIT and their comparison to the control group and then search for a viable efficacy marker in relation to patient symptoms.

Material and methods: AIT was administered in 22 patients allergic to grass pollen. Analysis of 15 selected transcript expression was performed in whole blood samples taken before AIT (sample A) and after reaching the maintenance dose (sample B). The control group included 25 healthy volunteers (sample C). The primary endpoint was Relative Quantification. The gene expression analysis was followed by clinical evaluation with the use of Allergy Control Score (ACS).

Results: Comparison between samples A and B of gene expression showed a significant increase in IFNG expression $(p=0.03)$. In relation to the control group, pretreatment samples from patients showed higher levels of AFAP1L1 $(p=0.006)$, COMMD8 $(p=0.001)$, PIK3CD $(p=0.027)$ and TWIST2 $(p=0.0003)$ in univariate analysis. A generalized linear regression model was built according to the Bayesian Information Criterion based on the IFNG, FCER1A and PCDHB10 expression pattern for prediction of the AIT outcome. The model showed a correlation in predicted and observed changes in ACS.

Conclusions: There is a significant change in the expression of IFNG during the build-up phase of AIT. The authors propose an in vitro model of AIT efficacy prediction for further validation.
\end{abstract}

Key words: allergic rhinitis, asthma, mRNA, allergen.

\section{Introduction}

Allergen-specific immunotherapy (AIT) is the core treatment in allergic rhinitis and asthma. AIT has been shown to influence numerous immunological reactions, including early desensitization, T-cell and B-cell tolerance and switch of antibody class from IgE to IgG. However, the mechanisms behind a successful AIT are not fully understood [1].

Early desensitization is a relatively fast process leading to the decrease in reactivity of mast cells and basophils, despite persisting presence of allergen specific lgE. This effect is specifically exposed in ultra-rush protocols when tolerance is usually developed in a few hours. Such prompt reaction results from expression of a histamine receptor $\mathrm{H} 2(\mathrm{H} 2 \mathrm{R})$ increase, leading to low FceR1 reactivity [2]. Further, H2R may stimulate Th1 response and modulate dendritic cells (DCs) reactions [3].

The effect on T cells during AIT is described as a switch from Th2 to Th1 pathway. Its most possible mechanism includes the induction of $T$ regulatory cells (Treg), which correlates with the clinical response in AIT. The transcription factor - FOXP3 may be helpful in Treg activation assessment [4-6]. Further, the levels of Treg products, such as interleukin-10 (IL-10) and transform-

Address for correspondence: Jan Romantowski PhD, Department of Allergology, Medical University of Gdansk, 7 Debinki St, 80-952 Gdansk, Poland, phone: +48 5834925 26, fax: +48 58349 16 25, e-mail: jromant@gumed.edu.pl Received: 13.01 .2020 , accepted: 1.04 .2020$. 
ing growth factor- $\beta$ (TGF- $\beta$ ), change during AIT $[1,7,8]$. The role of IL-10 is complex. It promotes Th2 response but also suppresses inflammation and nuclear factor- $\kappa \mathrm{B}$ (NF- $\kappa$ B) activation $[9,10]$. Although Tregs seem to play a crucial role in the development of allergen tolerance, their induction is highly regulated by DCs. These cells are responsible for cytokine environment in which naïve $T$ cells maturate. High stimulation of T cells through tolllike receptors (TLR) and DCs with proinflammatory cytokines (IL-6, TLR4, TLR8) breaks T cell allergen tolerance. This may suggest DC being the central point in AIT effect $[1,11,12]$.

The initial theory on AIT mechanisms focused on antibody class switch from slgE to slgG4 [13]. With no ability to activate FceRI on mast cells, slgG was suspected to play a protective role by binding the allergen. $\mathrm{B}$ regulatory cells (Breg) producing IL-10 are partially responsible for this mechanism. Although slgE levels do not correlate with clinical efficiency of AIT, still slgE/slgG4 ratio is used to monitor the immune system response to AIT [1, 14-17].

Until now no dominant mechanism or marker of AIT efficiency has been confirmed. Genetic studies seem to be a promising method to determine relations between gene expression levels and treatment outcome. The determination of the gene expression level using quantitative mRNA measurement with reverse transcription polymerase chain reaction is a preferred method to identify marginal changes in mRNA levels which correspond with cytokine levels or transcription factors. This method enables the analysis of cytokines which are usually expressed in low levels $[18,19]$.

\section{Aim}

The aim of this study was to analyse the expression of selected genes during the initial phase of AIT at two time points together with comparison to the control group. Relating the results to patients, symptoms severity would lead to the potential marker of treatment efficacy. By this analysis, we hope to be able to predict the outcome of AIT already at the early stage of the treatment. A whole genome study conducted by Niedoszytko et al. on patients starting AIT with wasp venom revealed 18 transcripts that change their expression with a correlation with clinical efficacy. Out of those genes, AFAP1L1, CLDN1, COMMD8, PCDHB10, PRLR, and TWIST2 were selected by the authors as those with the highest expression changes during treatment [20]. The clinical manifestation of wasp venom allergy is different, though the mechanism of tolerance development may be similar to AIT in allergic rhinitis. Following Pevec et al. results, FOXP3, GATA3, INPP5, TBX21, SYK and PIK3CD were selected as those changing the expression during AIT with house dust mite allergens [21]. Although FCER1A expression difference was not significant in this study $(p=0.07)$ Celesnik et al. showed otherwise [22]. Additionally, IFNG was chosen according to an early study by Varney et al. on patients receiving grass pollen AIT [23]. Finally TGFB was chosen due to its newly discovered impact on AIT well described by Akdis and Akdis [13]. The initial choice was strengthened by additional studies by Bonvalet et al. and Zheng et al. [22, 24, 25]. This search for a clinically useful biomarker addresses the needs implied by two main allergy societies: the American Academy of Allergy, Asthma \& Immunology and the European Academy of Allergy and Clinical Immunology. It is important to establish how a patient responds to the therapy as early as possible to avoid 3-year treatment in no responders and strengthen the compliance in responders. The golden standard, i.e. inhaled provocation is both troublesome and costly, thus it is not the best choice for clinical use. The biomarkers suggested to date, for instance specific IgE level, IgE/lgG4 ratio, basophil activation test, serum IgE inhibitory activity, Th1, Th2, Treg although promising have failed so far to give consistent results or still await proper validation in replicated trials [26].

\section{Material and methods}

The study patient group included 22 consecutive patients allergic to grass pollen ( 6 women and 16 men) with diagnosis of seasonal allergic rhinitis. The severity was assessed according to the Allergic Rhinitis and its Impact on Asthma guidelines (Table 1) [27]. All patients were recruited at the start of 3-year grass pollen subcutaneous AIT following the conventional protocol. The mean age of participants was 31 years (range: 18-41). All patients were diagnosed with allergic rhinitis and 15 (68\%) were additionally asthmatic (with a level of treatment according to Global Initiative for Asthma guidelines - Table 1) [28]. The most prevalent comorbidities were hypertension (3 patients) and smoking addiction (3 patients). Written, informed consent was obtained on enrolment. Blood samples were collected (using Tempus ${ }^{\text {TM }}$ blood RNA tube; Applied Biosystems ${ }^{\circledR}$ ) at two different time points: before starting the AIT and after reaching the maintenance dose (sample A and B, respectively). Blood collection before AIT was performed between August and May of the following year, which is outside the grass pollen season in northern Poland. The mean time between sample $A$ and $B$ collection was 138 days. Samples $C$ were obtained at the same time as samples A from 25 healthy donors with a negative allergy history and negative grass pollen specific lgE. All patients were evaluated for clinical treatment efficacy using the Allergy Control Score (ACS) based on non-commercial agreement with Allergo-Pharma. To assess symptoms during two consecutive pollen seasons the patients underwent ACS scoring before AIT and after 1 year of treatment [29]. The patient characteristics are presented in Table 1.

All blood samples were stored at $-80^{\circ} \mathrm{C}$ before processing. Isolated mRNA was used to perform reverse 
Table 1. Patient characteristics. Severity assessed according to ARIA guidelines

\begin{tabular}{|c|c|c|c|c|c|c|c|c|c|c|c|c|c|c|c|}
\hline 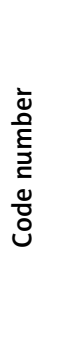 & 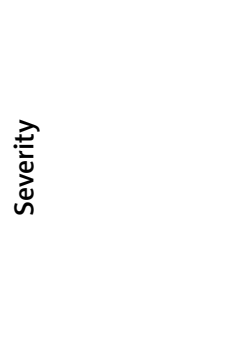 & $\overrightarrow{\tilde{u}}$ & $\frac{N}{\tilde{z}}$ & $\stackrel{\text { 品 }}{\alpha}$ & 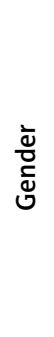 & 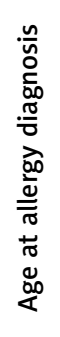 & 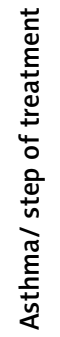 & 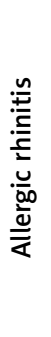 & 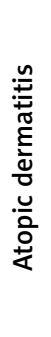 & 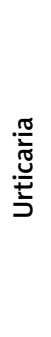 & 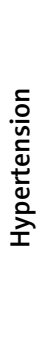 & 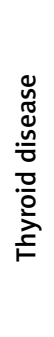 & 总 & 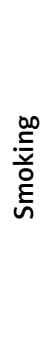 & 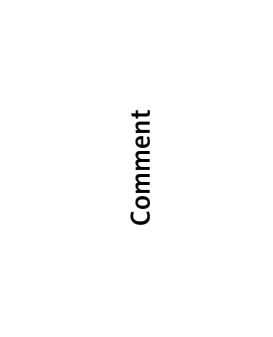 \\
\hline 1 & Mild & 20 & 16 & 40 & $M$ & 10 & $1 / 1$ & 1 & 0 & 0 & 0 & 0 & 0 & 0 & \\
\hline 2 & Mild & 12 & 11 & 46 & $M$ & 41 & 0 & 1 & 0 & 0 & 1 & 0 & 0 & 0 & \\
\hline 3 & Moderate/severe & 17 & 8 & 25 & M & 22 & 0 & 1 & 0 & 0 & 0 & 0 & 0 & 0 & \\
\hline 4 & Moderate/severe & 20 & 18 & 39 & M & 15 & $1 / 1$ & 1 & 0 & 0 & 0 & 0 & 0 & 0 & \\
\hline 5 & Moderate/severe & 34 & 27 & 20 & $M$ & 7 & $1 / 3$ & 1 & 0 & 0 & 0 & 0 & 0 & 0 & \\
\hline 6 & Moderate/severe & 17 & 25 & 37 & M & 27 & 0 & 1 & 0 & 0 & 1 & 0 & 0 & 0 & \\
\hline 7 & Moderate/severe & 25 & 13 & 29 & $\mathrm{~K}$ & 18 & $1 / 2$ & 1 & 0 & 0 & 0 & 0 & 0 & 0 & \\
\hline 8 & Mild & 9 & 14 & 24 & M & 14 & 0 & 1 & 0 & 0 & 0 & 0 & 0 & 0 & \\
\hline 9 & Moderate/severe & 33 & & 18 & $\mathrm{~K}$ & 9 & $1 / 3$ & 1 & 0 & 0 & 0 & 0 & 0 & 0 & Denied second survey \\
\hline 10 & Mild & 23 & 19 & 32 & M & 10 & $1 / 3$ & 1 & 0 & 0 & 0 & 0 & 0 & 0 & \\
\hline 11 & Moderate/severe & 37 & 38 & 19 & $\mathrm{~K}$ & 5 & $1 / 3$ & 1 & 0 & 0 & 0 & 0 & 0 & 0 & \\
\hline 12 & Moderate/severe & 25 & & 28 & $\mathrm{~K}$ & 23 & $1 / 3$ & 1 & 0 & 0 & 0 & 0 & 0 & 0 & Denied second survey \\
\hline 13 & Moderate/severe & 33 & 31 & 37 & M & 17 & $1 / 3$ & 1 & 0 & 0 & 0 & 0 & 0 & 1 & \\
\hline 14 & Moderate/severe & 14 & & 28 & M & & $1 / 3$ & 1 & 0 & 0 & 0 & 0 & 0 & 0 & AIT interrupted \\
\hline 15 & Moderate/severe & 11 & & 40 & M & 30 & 0 & 1 & 0 & 0 & 0 & 0 & 0 & 0 & Denied second survey \\
\hline 16 & Mild & 15 & 6 & 28 & M & 7 & 0 & 1 & 0 & 0 & 0 & 0 & 0 & 0 & \\
\hline 17 & Moderate/severe & 20 & 13 & 45 & $M$ & 30 & $1 / 1$ & 1 & 0 & 0 & 0 & 0 & 0 & 0 & \\
\hline 18 & Moderate/severe & 8 & 10 & 27 & M & & 0 & 1 & 0 & 0 & 0 & 0 & 0 & 1 & \\
\hline 19 & Moderate/severe & 25 & 9 & 21 & $\mathrm{~K}$ & & $1 / 3$ & 1 & 0 & 0 & 0 & 0 & 1 & 0 & \\
\hline 20 & Moderate/severe & 25 & 17 & 36 & $M$ & 22 & 0 & 1 & 0 & 0 & 0 & 0 & 0 & 1 & \\
\hline 21 & Moderate/severe & 16 & 15 & 38 & $\mathrm{~K}$ & 20 & $1 / 1$ & 1 & 0 & 0 & 0 & 0 & 0 & 0 & \\
\hline 22 & Moderate/severe & 25 & 17 & 38 & $M$ & 3 & $1 / 1$ & 1 & 0 & 0 & 1 & 0 & 1 & 0 & \\
\hline
\end{tabular}

ACS - allergy control score. The results of ACS are presented before treatment and after 1 year of AIT (1 and 2 respectively), GERD - gastroesophageal reflux disease.

transcription with Tempus ${ }^{\text {TM }}$ Spin RNA Isolation Kit (Applied Biosystems ${ }^{\oplus}$ ). Isolated total mRNA was transferred to perform reverse transcription with High Capacity cDNA Reverse Transcription Kit (Applied Biosystems ${ }^{\circledast}$ ) in Thermal Cycler 9700 (Applied Biosystems ${ }^{\oplus}$ ). 450 ng Universal Human Reference RNA (Agilent Technologies) was used as a control. The cDNA product was then transferred to TaqMan Array Micro Fluidic Cards (Applied Biosystems ${ }^{\circledR}$ ) for PCR in 7900HT Real Time Fast PCR System (Applied Biosystems ${ }^{\circledast}$ ). The microfluidic cards had 16 probes placed by the manufacturer for detection of the following genes: AFAP1L1, CLDN1, COMMD8, FCER1A, FOXP3, GATA3, IFNG, INPP5D, PCDHB10, PIK3CD, PRLR, SYK, TBX21, TGFB, TWIST2, and 18S. 18 S ribosomal rRNA was used as a housekeeping gene for normalization. All above-mentioned laboratory procedures were performed according to the manufacturer's instructions.

The result of PCR was expressed as a number of cycles until cDNA concentration crossed set threshold. The number was then normalized with 185 and referred to calibrator 450 ng Universal Human Reference RNA (Agilent Technologies). Primary endpoint was Relative Quantification (RQ) calculated according to MIQE guidelines [30].

The study was approved by the appropriate Ethics Commission at the Medical University of Gdansk.

\section{Statistical analysis}

Statistical analysis was performed with Statistica 12 software (StatSoft Tulsa, USA) on non-commercial licence of the Medical University of Gdansk. The descriptive 
statistics as: mean, median, standard deviation, lower and upper quartile and their $95 \%$ confidence intervals (95\% Cl) were calculated for each variable within each group. The Lilliefors test was applied to verify the hypothesis on normality of gene expression distribution within each subpopulation while F test was used to check on the variance homogeneity. Depending on these results, the parametric (paired t test) or nonparametric test (MannWhitney $U$ test) was performed to verify the statement on mean/median value equality between subpopulations. In case of the paired data, the test used was one sample $t$ test or Wilcoxon rank test. The statistical testing was accompanied by the estimation of the effect size done by Cohen's d statistics. Finally, the linear regression model for prediction of AIT clinical success was built with the support of the forward feature selection procedure and Bayesian Information Criterion (BIC) for model selection.

\section{Results}

A significant change in expression between samples $A$ and $B$ was found in the IFNG gene (paired $t$ test, $p=0.0243$, medium effect, Cohen's $d=0.5174$ ) and additionally a change nearing significance for TBX21 (Wilcoxon tests $p=0.0883$; medium effect, Cohen's $d=$ 0.5266). The mean expression changes in IFNG during AIT (samples A vs. B vs. C) are demonstrated in Figure 1. Mann Whitney test analysis was performed for $22 \mathrm{pa}$ tients and 25 healthy controls and revealed a significant difference between sample $A$ and $C$ in AFAP1L1, and PIK3CD (Table 2). A significant difference was observed

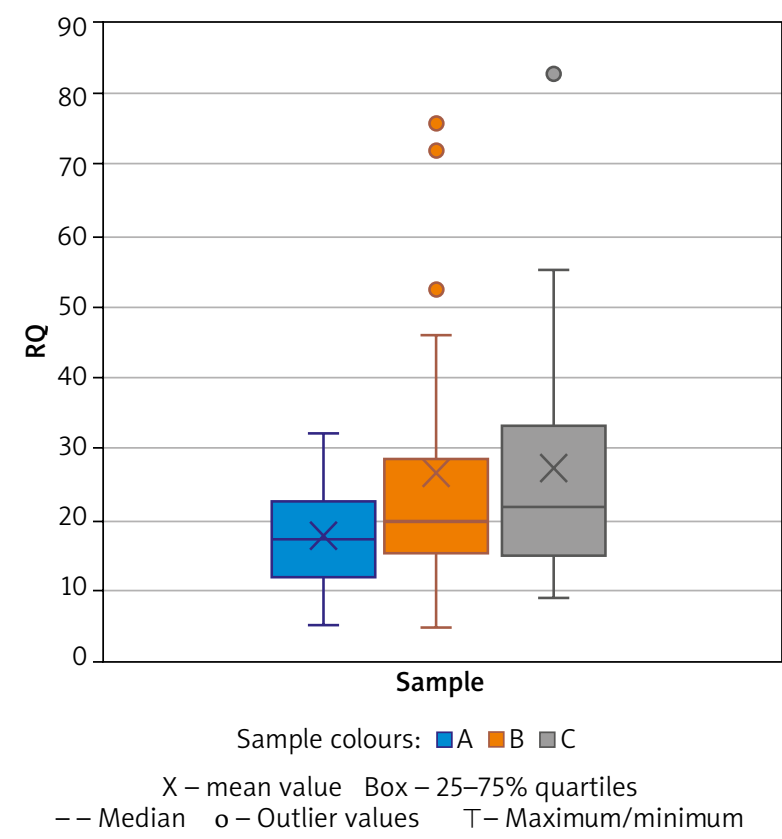

Figure 1. Expression of IFNG shown by Relative Quantification (RQ). Comparison of samples A (before treatment) vs. $B$ (during treatment) and C (healthy controls) in COMMD8 and TWIST2 using the parametric test. The highest significance was observed for COMMD8 ( $t$ test, $p=0.0014$, large effect, Cohen's $d=0.9950$ ).

In total 18 patients had completed the two ACS assessments (4 were lost during follow-up). ACS decreased significantly during the first year of treatment from average 21.17 to 17.06 points (paired test $p=0.0102$ ). The final linear model predicts the 1 year change of ACS based on the expression of three genes: IFNG, FCER1A, PCDHB10 ( $p=0.00785$, Table 3$)$. The algorithm properly predicted the outcome of AIT (lowering or increasing of ACS) in 16 out of 18 cases based on gene expression in sample A (89\%). The comparison of observed and predicted ACS change is presented in Figure 2. Regression analysis revealed correlation between predicted and observed ACS change (Pearson's $r=0.7484, p=0.0004$ ). The interaction network presented in Figure 3 (obtained from "STRING: Functional protein association networks" [31]) demonstrates our predictors, chosen as the most representative and the most informative to the problem. Note that they belong to the separate gene clusters.

\section{Discussion}

The present study comprising patients receiving grass pollen AIT included expression analysis of selected 15 genes. Presented results are partially convergent with those published to date. The population of patients allergic to grass pollen was only assessed by one major study so far (with a similar method). It was conducted in 1993, included 40 patients allergic to grass pollen and showed an increase in IFNG and IL-2 expression during treatment [23]. In a similar study including 39 patients administered house dust mite AIT showed a treatment-induced increase in expression of FOXP3, SHIP and TBX21, and a decrease of GATA3, SYK and PIK3CD [21]. Other study demonstrated that several weeks of insect venom AIT resulted in a significant decrease of mastocyte receptor FCER1 [22]. A FOXP3 expression increase was also observed in a study in 18 patients receiving house dust mite AIT [25].

The above results are not fully consistent with other analyses. For example, in the double blind placebo controlled study on 89 patients administered Sublingual Immunotherapy (SLIT) there was no expression change of FOXP3, GATA3, IFNG, IL-17, IL-10, and IL-4 and there was only a transient expression increase in TBX21 and TGFB [24].

In our study, the change in IFNG was the only gene transcript showing a significant increase during treatment. TBX21 showed a medium level of effect size similar to IFNG, but $p$-value (0.08) close to the statistical significance.

Interferon g binding receptors, IFNGR1 and IFNGR2, activate JAK and STAT kinase pathways. This contributes to differentiation and proliferation of B cells, suppression of IgE production and switch to Th1 instead of Th2 in re- 
Table 2. Comparison results of gene expression for samples A vs. B and A vs. C

\begin{tabular}{|c|c|c|c|c|c|c|c|}
\hline Gene & $\begin{array}{c}\text { Sample } \\
\text { A-B, } n\end{array}$ & $P$-value & Cohen's $d$ & $\begin{array}{c}\text { Sample } \\
\text { A, } n\end{array}$ & $\begin{array}{c}\text { Sample } \\
\text { C, } n\end{array}$ & $P$-value & Cohen's $d$ \\
\hline AFAP1L1 & 22 & $0.8696^{1}$ & 0.0354 & 22 & 25 & $0.0102^{2}$ & 0.5078 \\
\hline CLDN1 & 22 & $0.7826^{2}$ & 0.1103 & 22 & 25 & $0.1122^{2}$ & 0.4613 \\
\hline COMMD8 & 22 & $0.2361^{1}$ & 0.2600 & 22 & 25 & $0.0014^{1}$ & 0.9950 \\
\hline FCER1A & 22 & $0.5481^{2}$ & 0.1374 & 22 & 25 & $0.1222^{2}$ & 0.3873 \\
\hline FOXP3 & 22 & $0.9353^{2}$ & 0.1490 & 22 & 25 & $0.3879^{2}$ & 0.0590 \\
\hline GATA3 & 22 & $0.5597^{1}$ & 0.1264 & 22 & 25 & $0.2540^{2}$ & 0.0192 \\
\hline IFNG & 22 & $0.0243^{1}$ & 0.5174 & 22 & 25 & $0.1171^{2}$ & 0.6484 \\
\hline INPP5D & 22 & $0.6378^{2}$ & 0.1265 & 22 & 25 & $0.8395^{2}$ & 0.1384 \\
\hline PCDHB10 & 22 & $0.9870^{2}$ & 0.0645 & 22 & 25 & $0.2816^{2}$ & 0.3038 \\
\hline$P I K 3 C D$ & 22 & $0.9095^{2}$ & 0.1424 & 22 & 25 & $0.0376^{2}$ & 0.5606 \\
\hline$P R L R$ & 22 & $0.2913^{2}$ & 0.2644 & 22 & 25 & $0.2913^{2}$ & 0.0943 \\
\hline$S Y K$ & 22 & $0.7376^{1}$ & 0.0724 & 22 & 25 & $0.3215^{2}$ & 0.0567 \\
\hline$T B X 21$ & 22 & $0.0883^{2}$ & 0.5266 & 22 & 25 & $0.3601^{1}$ & 0.2703 \\
\hline$T G F B$ & 22 & $0.7633^{1}$ & 0.0650 & 22 & 25 & $0.3215^{2}$ & 0.0349 \\
\hline TWIST2 & 22 & $0.6389^{2}$ & 0.1726 & 22 & 25 & $0.0002^{1}$ & 0.7461 \\
\hline
\end{tabular}

${ }^{1}$ Parametric test; ${ }^{2}$ nonparametric test.

Table 3. Parameters of the final linear predictive model for 1 year ACS changes: $d A C S=f(F C E R 1 A$, IFNG, PCDHB10) obtained for 18 observations; 14 error degrees of freedom F-statistic vs. constant model: 5.94, $p$-value $=0.00785$

\begin{tabular}{lcccc}
\hline Gene & Coefficient estimate & Standard error & tstat & P-value \\
\hline Intercept) & -0.5027 & 3.2511 & -0.1546 & 0.8793 \\
\hline FCER1A & -0.2225 & 0.1209 & -1.8404 & 0.0870 \\
\hline IFNG & 0.4460 & 0.1724 & 2.5873 & 0.0215 \\
\hline PCDHB10 & -4.5377 & 1.1831 & -3.8355 & 0.0018 \\
\hline
\end{tabular}

sponse to immunotherapy. This mechanism has also an impact on dendritic cell maturation [32-39]. Therefore, IFNG has a multilevel influence on the immune system. However, due to its connection with lymphocytes, its impact on desensitization may be limited.

TBX21 is a transcription factor responsible for maturation of naïve T cells towards Th1. It also enhances IFNG expression creating a synergistic loop [40, 41]. A polymorphism of TBX21 resulting in its lower expression stimulates Th2 cytokine production and aggravates asthma symptoms [42]. GATA3 is a transcription factor with an important role in Th2 cell maturation and an opposite function to TBX21. Its high expression may contribute to development of allergy [43, 44]. However in our study, the expression of GATA3 and TBX21 did not change significantly during treatment $[21,24]$.

In our study the comparison between patients allergic to grass pollen prior to treatment and healthy controls revealed a significantly different expression of PIK3CD, COMMD8, TWIST2 and AFAP1L1. This observation suggests resemblance of mechanisms in insect venom and grass pollen sensitization [45].

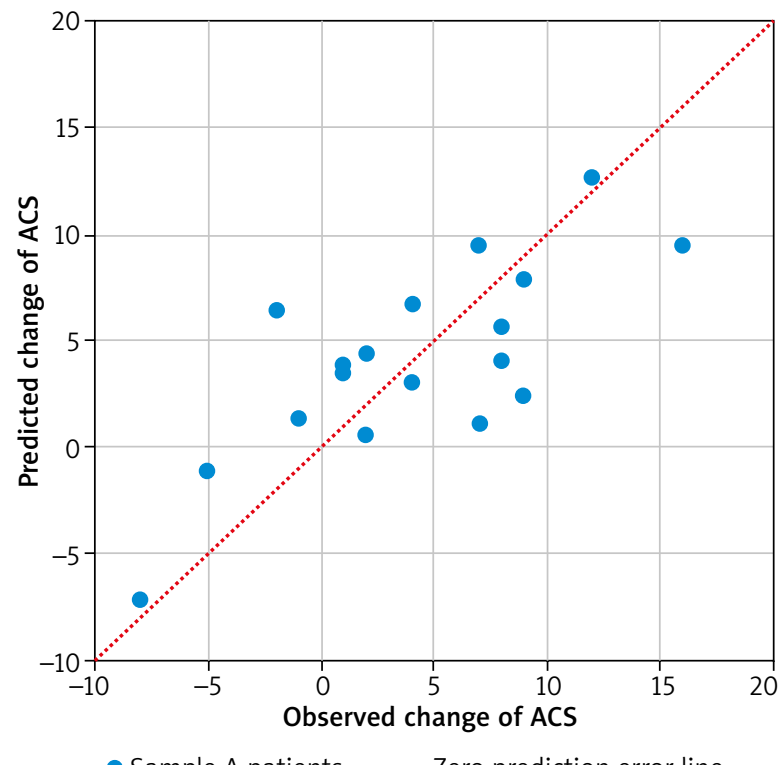

- Sample A patients ........ Zero prediction error line

Figure 2. Prediction model of the changes of ACS level (before treatment and after 1 year (first pollen season)) in patients based on sample A IFNG, FCER1A, PCDHB10 expression (adjusted $R^{2}=0.46579$ ) 


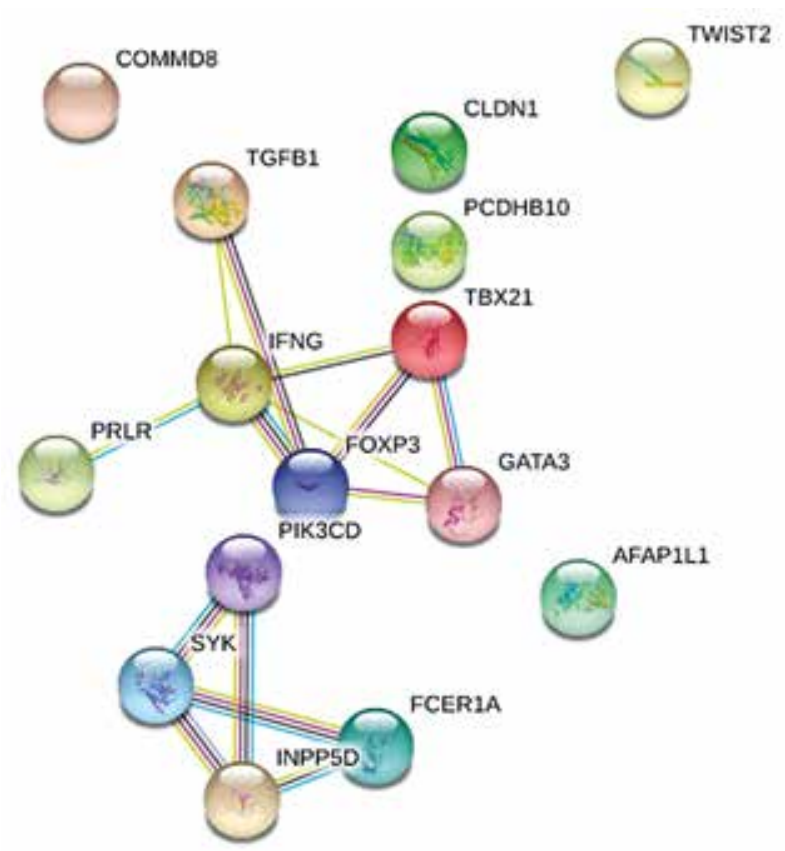

Figure 3. The interaction network among the analysed 15 genes

The PIK3CD gene codes delta subunit of phosphoinositide 3-kinase. These enzymes phosphorylate inositol lipids and are involved in the immune response. Their expression is usually increased in leukocytes and resembles signalling through B cell receptors. They are also postulated to play an important role in mastocyte activation $[46,47]$. PIK3CD expression influences asthma and allergic rhinitis. Idelalis$i b$, an inhibitor of $110 \delta$ subunit, is currently investigated in a clinical trial on patients with allergic rhinitis [48].

Some data indicate that COMMD8 induces release of $\mathrm{NF}-\kappa \mathrm{B}$ and enables its transcription factor function in nucleus [43-45]. NF- $\kappa B$ was shown to play an important role in asthma, chronic inflammation by stimulating (among others) DCs to cytokine production and modulating the IFNG influence on Th2 to Th1 T cell switch [49]. Finally, NF-кB increases Treg population and maturation - an effect demonstrated in the clinical trial including 20 children administered immunotherapy [50].

The function of TWIST2, a transcription factor, remains unknown. Its lack increases a proinflammatory response of NF- $\kappa \mathrm{B}$ and decreases the function of IFNG. TWIST2 has probably also an anti-inflammatory activity and its low expression in patients may be due to allergic inflammation. Similarly to other transcription factors, its expression is low and probably susceptible to many stimuli [51, 52].

AFAP1L1, in turn, is a gene associated with intracellular transport. AFAP1 proteins are responsible for actin binding and podosome, as well as invadopodia forming, which enables cell migration and interactions with environment. AFAP1L1 expression changes may be induced by immuno- therapy due to non-specific changes in the immune system activation $[53,54]$.

Primary hypothesis highlighted importance of $\mathrm{T}$ regulatory cells (Treg) in AIT, even though FOXP3, a marker of T-cell proliferation and activation did not change significantly during treatment and was not different in healthy controls. Such discrepancies have been reported earlier by other authors. However, the change of IFNG expression also contributes to Treg suppressive activity as presence of IFNG is required for their function. Still, it is unknown why a change in FOXP3 expression is inconsistent [20, 24, 55].

The assumption for the linear prediction regression algorithm was that gene expression from samples A may predict AIT outcome. With the help of the Bayesian Information Criterion, chosen genes (IFNG, FCER1A, PCDHB10) may be used in the future to predict AIT efficacy in certain patients and support decision towards continuing such long and difficult therapy as AIT. Although FCER1A, PCDHB10 by themselves did not reveal statistical significance in initial comparisons; together with IFNG managed to build an algorithm. Eighty-nine percent of successful prediction followed by confirmed correlation based on initial sample A may be promising. The authors are aware that further prospective, multi-centre validation is required for this model to enter clinical application. In specific immunotherapy similar models were introduced for patients allergic to insect venom. Niedoszytko et al. built a Naïve Bayes model with 18 genes differentiating, with $82 \%$ accuracy, patients tolerant to insect venom after treatment and those prone to anaphylaxis despite treatment. Of note, this model also included among others PCDHB10 and IFNG [45].

Unfortunately, in efficacy assessment four out of $22 \mathrm{pa}$ tients did not complete the second ACS survey. Although their characteristics did not differ significantly from the others, still, the denial of three of them may be due to the lack of compliance or other logistic reasons. The final patient interrupting AIT prematurely shows how difficult AIT is in terms of maintenance. These dropouts support the need for early stage biomarkers that would enhance the patients' motivation.

\section{Conclusions}

The AFAP1L1, COMMD8, PIK3CD and TWIST2 genes have a different expression in patients allergic to grass pollen compared to healthy controls. IFNG changes its expression during specific immunotherapy. A generalized linear regression algorithm based on IFNG, FCER1A, PCDHB10 expression may predict the outcome of AIT which requires prospective validation in double blind placebo controlled trials.

\section{Acknowledgments}

The study was funded by the Medical University of Gdansk: task number MN 01-0169/08. 
Publication costs were financed with Allergology Department statutory funding in Medical University of Gdansk ST554.

\section{Conflict of interest}

The authors declare no conflict of interest.

\section{References}

1. Jutel M, Agache I, Bonini S, et al. International Consensus on Allergen Immunotherapy II: Mechanisms, standardization, and pharmacoeconomics. J Allergy Clin. Immunol 2016; 137: 358-68.

2. Novak N, Mete N, Bussmann C, et al. Early suppression of basophil activation during allergen-specific immunotherapy by histamine receptor 2. J Allergy Clin Immunol 2012; 130: 1153-8.

3. O'Mahony L, Akdis M, Akdis CA. Regulation of the immune response and inflammation by histamine and histamine receptors. J Allergy Clin Immunol 2011; 128: 1153-62.

4. Akdis M, Akdis CA. Mechanisms of allergen-specific immunotherapy: multiple suppressor factors at work in immune tolerance to allergens. J Allergy Clin Immunol 2017; 133: 621-31.

5. Jutel M, Akdis CA. Immunological mechanisms of allergenspecific immunotherapy. Allergy 2011; 66: 725-32.

6. Suárez-Fueyo A, Ramos T, Galán A, et al. Grass tablet sublingual immunotherapy downregulates the Th2 cytokine response followed by regulatory T-cell generation. J Allergy Clin Immunol 2014; 133: 130-8.e1-2.

7. Möbs C, Ipsen H, Mayer L, et al. Birch pollen immunotherapy results in long-term loss of Bet $v 1$-specific Th2 responses, transient TR1 activation, and synthesis of IgE-blocking antibodies. J Allergy Clin Immunol 2017; 130: 1108-16.e6.

8. Radulovic S, Jacobson MR, Durham SR, et al. Grass pollen immunotherapy induces Foxp3-expressing CD4+CD25+ cells in the nasal mucosa. J Allergy Clin Immunol 2017; 121: 146772.e1.

9. Moore KW, de Waal Malefyt R, Coffman RL, et al. Interleukin-10 and the interleukin-10 receptor. Annu Rev Immunol 2001; 19: 683-765.

10. Moniuszko M, Grubczak K, Kowal K, et al. Development of asthmatic response upon bronchial allergen challenge is associated with dynamic changes of interleukin-10-producing and interleukin-10-responding CD4+ T cells. Inflammation 2014; 37: 1945-56.

11. Palomares O, Martin-Fontecha M, Lauener R, et al. Regulatory $T$ cells and immune regulation of allergic diseases: roles of IL-10 and TGF-beta. Genes Immun 2014; 15: 511-20.

12. Kücüksezer UC, Palomares O, Rückert B, et al. Triggering of specific Toll-like receptors and proinflammatory cytokines breaks allergen-specific T-cell tolerance in human tonsils and peripheral blood. J Allergy Clin Immunol 2013; 131: 87585.e9.

13. Akdis CA, Akdis M. Mechanisms of allergen-specific immunotherapy and immune tolerance to allergens. World Allergy Organ J 2015; 8: 17.

14. Meiler F, Klunker S, Zimmermann M, et al. Distinct regulation of IgE, IgG4 and IgA by T regulatory cells and toll-like receptors. Allergy 2008; 63: 1455-63.

15. Stanic B, van de Veen W, Wirz OF, et al. IL-10-overexpressing $B$ cells regulate innate and adaptive immune responses. J Allergy Clin Immunol 2017; 135: 771-80.e8.
16. Shamji MH, Ljørring C, Francis JN, et al. Functional rather than immunoreactive levels of IgG4 correlate closely with clinical response to grass pollen immunotherapy. Allergy 2012; 67: 217-26.

17. Wachholz PA, Durham SR. Mechanisms of immunotherapy: IgG revisited. Curr Opin Allergy Clin Immunol 2004; 4: 313-8.

18. Fletcher HA, Owiafe $P$, Jeffries $D$, et al. Increased expression of mRNA encoding interleukin (IL)-4 and its splice variant IL-4delta2 in cells from contacts of Mycobacterium tuberculosis, in the absence of in vitro stimulation. Immunology 2004; 112: 669-73.

19. Demissie A, Abebe M, Aseffa A, et al. Healthy individuals that control a latent infection with Mycobacterium tuberculosis express high levels of Th1 cytokines and the IL-4 antagonist IL-4delta2. I Immunol 2004; 172: 6938-43.

20. Niedoszytko M, Bruinenberg M, de Monchy J, et al. Changes in gene expression caused by insect venom immunotherapy responsible for the long-term protection of insect venomallergic patients. Ann Allergy Asthma Immunol 2011; 106: 502-10.

21. Pevec B, Radulovic Pevec M, Stipic Markovic A, et al. House dust mite-specific immunotherapy alters the basal expression of T regulatory and Fc RI pathway genes. Int Arch Allergy Immunol 2012; 159: 287-96.

22. Celesnik N, Vesel T, Rijavec M, et al. Short-term venom immunotherapy induces desensitization of FcepsilonRI-mediated basophil response. Allergy 2012; 67: 1594-600.

23. Varney VA, Hamid QA, Gaga M, et al. Influence of grass pollen immunotherapy on cellular infiltration and cytokine mRNA expression during allergen-induced late-phase cutaneous responses. J Clin Invest 1993; 92: 644-51.

24. Bonvalet $\mathrm{M}$, Moussu H, Wambre E, et al. Allergen-specific CD4+ T cell responses in peripheral blood do not predict the early onset of clinical efficacy during grass pollen sublingual immunotherapy. Clin Exp Allergy 2012; 42: 1745-55.

25. Zheng R, Wu X, Huang X, et al. Gene expression pattern of Treg and TCR $V$ subfamily $T$ cells before and after specific immunotherapy in allergic rhinitis. J Transl Med 2014; 12: 24.

26. Shamji MH, Durham SR. Mechanisms of allergen immunotherapy for inhaled allergens and predictive biomarkers. J Allergy Clin Immunol 2017; 140: 1485-98.

27. Brozek JL, Bousquet J, Agache I, et al. Allergic Rhinitis and its Impact on Asthma (ARIA) guidelines-2016 revision. J Allergy Clin Immunol 2017; 140: 950-8.

28. Horak F, Doberer D, Eber E, et al. Diagnosis and management of asthma - Statement on the 2015 GINA Guidelines. Wien Klin Wochenschr 2016; 128: 541-54.

29. Hafner D, Reich K, Matricardi PM, et al. Prospective validation of "Allergy-Control-SCORE(TM)": a novel symptommedication score for clinical trials. Allergy 2011; 66: 629-36.

30. Bustin SA, Benes V, Garson JA, et al. The MIQE guidelines: minimum information for publication of quantitative realtime PCR experiments. Clin Chem 2009; 55: 611-22.

31. STRING: Functional protein association networks [Internet]. Available from: http://string-db.com/.

32. Sadir R, Forest E, Lortat-Jacob H. The heparan sulfate binding sequence of interferon-gamma increased the on rate of the interferon-gamma-interferon-gamma receptor complex formation. J Biol Chem 1998; 273: 10919-25.

33. Delneste $\mathrm{Y}$, Charbonnier P, Herbault N, et al. Interferon- $\gamma$ switches monocyte differentiation from dendritic cells to macrophages. Blood 2003; 101: 143-50. 
34. Senik A, Stefanos S, Kolb JP, et al. Enhancement of mouse natural killer cell activity by type II interferon. Ann Immunol 1980; 131: 349-61.

35. Coffman RL, Carty J. At T cell activity that enhances polyclonal IgE production and its inhibition by interferon- $\gamma$. I Immunol 1986; 136: 949-54.

36. Mond JJ, Finkelman FD, Sarma C, et al. Recombinant interferon- $\gamma$ inhibits the B cell proliferative response stimulated by soluble but not by Sepharose-bound anti-immunoglobulin antibody. J Immunol 1985; 135: 2513-7.

37. Leibson HJ, Gefter M, Zlotnik A, et al. Role of $\gamma$-interferon in antibody-producing responses. Nature 1984; 309: 799-801.

38. Rothermel CD, Rubin BY, Murray HW. $\gamma$-Interferon is the factor in lymphokine that activates human macrophages to inhibit intracellular Chlamydia psittaci replication. J Immunol 1983; 131: 2542-4.

39. Billiau A, Matthys P. Interferon-gamma: a historical perspective. Cytokine Growth Factor Rev 2009; 20: 97-113.

40. Zhu J, Jankovic D, Oler AJ, et al. The transcription factor T-bet is induced by multiple pathways and prevents an endogenous Th2 cell program during Th1 cell responses. Immunity 2012; 37: 660-73.

41. Leng RX, Pan HF, Liu J, et al. Evidence for genetic association of TBX21 and IFNG with systemic lupus erythematosus in a Chinese Han population. Sci Rep 2016; 6: 22081.

42. Sharma N, Jaiswal I, Mandal RK, et al. Genetic variation of TBX21 gene increases risk of asthma and its severity in Indian children. J Hum Genet 2014; 59: 437-43.

43. Sasaki T, Onodera A, Hosokawa H, et al. Genome-wide gene expression profiling revealed a critical role for GATA3 in the maintenance of the Th2 cell identity. PLoS One 2013; 8: e66468.

44. Abelius MS, Janefjord C, Ernerudh J, et al. The placental immune milieu is characterized by a Th2- and anti-inflammatory transcription profile, regardless of maternal allergy, and associates with neonatal immunity. Am J Reprod Immunol 2015; 73: 445-59.

45. Niedoszytko M, Bruinenberg M, de Monchy J, et al. Gene expression analysis in predicting the effectiveness of insect venom immunotherapy. J Allergy Clin Immunol 2010; 125: 1092-7.

46. Werner M, Hobeika E, Jumaa H. Role of PI3K in the generation and survival of B cells. Immunol Rev 2010; 237: 55-71.

47. Vanhaesebroeck B, Guillermet-Guibert J, Graupera M, et al. The emerging mechanisms of isoform-specific PI3K signalling. Nat Rev Mol Cell Biol 2010; 11: 329-41.

48. Horak F, Puri KD, Steiner BH, et al. Randomized phase 1 study of the phosphatidylinositol 3-kinase delta inhibitor idelalisib in patients with allergic rhinitis. J Allergy Clin Immunol 2016; 137: 1733-41.

49. Gerondakis S, Fulford TS, Messina NL, et al. NF-kappaB control of T cell development. Nat Immunol 2014; 15: 15-25.

50. Tsai YG, Chiou YL, Chien JW, et al. Induction of IL-10+ CD4+ CD25+ regulatory T cells with decreased NF-kappaB expression during immunotherapy. Pediatr Allergy Immunol 2010; 21: e166-73.

51. Sharif MN, Šošić D, Rothlin CV, et al. Twist mediates suppression of inflammation by type I IFNs and Axl. J Exp Med 2006; 203: 1891-901.

52. Sosic D, Richardson JA, Yu K, et al. Twist regulates cytokine gene expression through a negative feedback loop that represses NF-kappaB activity. Cell 2003; 112: 169-80.
53. Snyder BN, Cho Y, Qian Y, et al. AFAP1L1 is a novel adaptor protein of the AFAP family that interacts with cortactin and localizes to invadosomes. Eur J Cell Biol 2011; 90: 376-89.

54. Murphy DA, Courtneidge SA. The "ins" and "outs" of podosomes and invadopodia: characteristics, formation and function. Nat Rev Mol Cell Biol 2011; 12: 413-26.

55. Wood KJ, Sawitzki B. Interferon $\gamma$ : a crucial role in the function of induced regulatory T cells in vivo. Trends Immunol 2006; 27: 183-7. 